\title{
BIG BANG NUCLEOSYNTHESIS
}

\author{
D.N. SCHRAMM \\ The University of Chicago \\ 5640 S. Ellis Avenue \\ Chicago, IL 60637, USA
}

\begin{abstract}
Big Bang Nucleosynthesis (BBN) is on the verge of undergoing a transformation now that extragalactic deuterium is being measured. Previously, the emphasis was on demonstrating the concordance of the Big Bang Nucleosynthesis model with the abundances of the light isotopes extrapolated back to their primordial values using stellar and Galactic evolution theories. Once the primordial deuterium abundance is converged upon, the nature of the field will shift to using the much more precise primordial $\mathrm{D} / \mathrm{H}$ to constrain the more flexible stellar and Galactic evolution models (although the question of potential systematic error in ${ }^{4} \mathrm{He}$ abundance determinations remains open). The remarkable success of the theory to date in establishing the concordance has led to the very robust conclusion of BBN regarding the baryon density. The BBN constraints on the cosmological baryon density are reviewed and demonstrate that the bulk of the baryons are dark and also that the bulk of the matter in the universe is non-baryonic. Comparison of baryonic density arguments from Lyman- $\alpha$ clouds, $\mathrm{x}$-ray gas in clusters, the Sunyaev-Zeldovich effect, and the microwave anisotropy are made and shown to be consistent with the BBN value.
\end{abstract}

\section{Introduction}

Big Bang Nucleosynthesis and the study of the light element abundances is undergoing a major transformation. The bottom line remains: primordial nucleosynthesis has joined the Hubble expansion and the microwave background radiation as one of the three pillars of Big Bang cosmology. Of the three, Big Bang Nucleosynthesis (BBN) probes the universe to far earlier times $(\sim 1 \mathrm{sec})$ than the other two and led to the interplay of cosmology with nuclear and particle physics. Furthermore, since the Hubble expansion 
is also part of alternative cosmologies such as the steady state, it is BBN and the microwave background that really drive us to the conclusion that the early universe was hot and dense. The new extragalactic deuterium observations not only cement this picture and give added convergence on a value of the baryon density, $\Omega_{b}$, they also enable BBN to become a constraint on stellar and Galactic evolution scenarios. It is this latter point that is the core of the transformation. Furthermore, new alternative methods of estimating the cosmic baryon density are now coming into use and are independently confirming the BBN prediction.

The current reviews will draw heavily on the recent reviews by Schramm (1997) in the Proceedings of the National Academy of Sciences and the proceedings of the Symposium on Light Element Synthesis held in Bern, Switzerland.

\section{Overview}

Although the extragalactic $\mathrm{D} / \mathrm{H}$ observations have naturally attracted the most attention, it should not be forgotten that there are also recent heroic observations of ${ }^{6} \mathrm{Li}, \mathrm{Be}$ and $\mathrm{B}$, as well as ${ }^{3} \mathrm{He}$ and new ${ }^{4} \mathrm{He}$ determinations. Let us now briefly review the history, with special emphasis on the remarkable agreement of the observed light element abundances with the calculations. This agreement works only if the baryon density is well below the cosmological critical value. We will also note how a convergence on extragalctic $\mathrm{D} / \mathrm{H}$ will enable powerful new constraints on stellar and Galactic evolution.

It should be noted that there is a symbiotic connection between BBN and the $3 \mathrm{~K}$ background dating back to Gamow and his associates, Alpher and Herman. The initial BBN calculations of Gamow's group (Alpher et al., 1948) assumed pure neutrons as an initial condition and thus were not particularly accurate, but their inaccuracies had little effect on the group's predictions for a background radiation.

Once Hayashi (1950) recognized the role of neutron-proton equilibration, the framework for BBN calculations themselves has not varied significantly. The work of Alpher, Follin and Herman (1953) and Tayler and Hoyle (1964), preceeding the discovery of the $3 \mathrm{~K}$ background, and of Peebles (1966) and Wagoner, Fowler and Hoyle (1967), immediately following the discovery, and the more recent work of our group of collaborators (Copi et al., 1997; Copi et al., 1994; Walker et al., 1991; Olive et al., 1990; Schramm and Wagoner, 1977; Olive et al., 1981; Yang et al., 1984; Kawano et al., 1988) all do essentially the same basic calculation, the results of which are shown in Figure 1.

As far as the calculation itself goes, solving the reaction network is rel- 


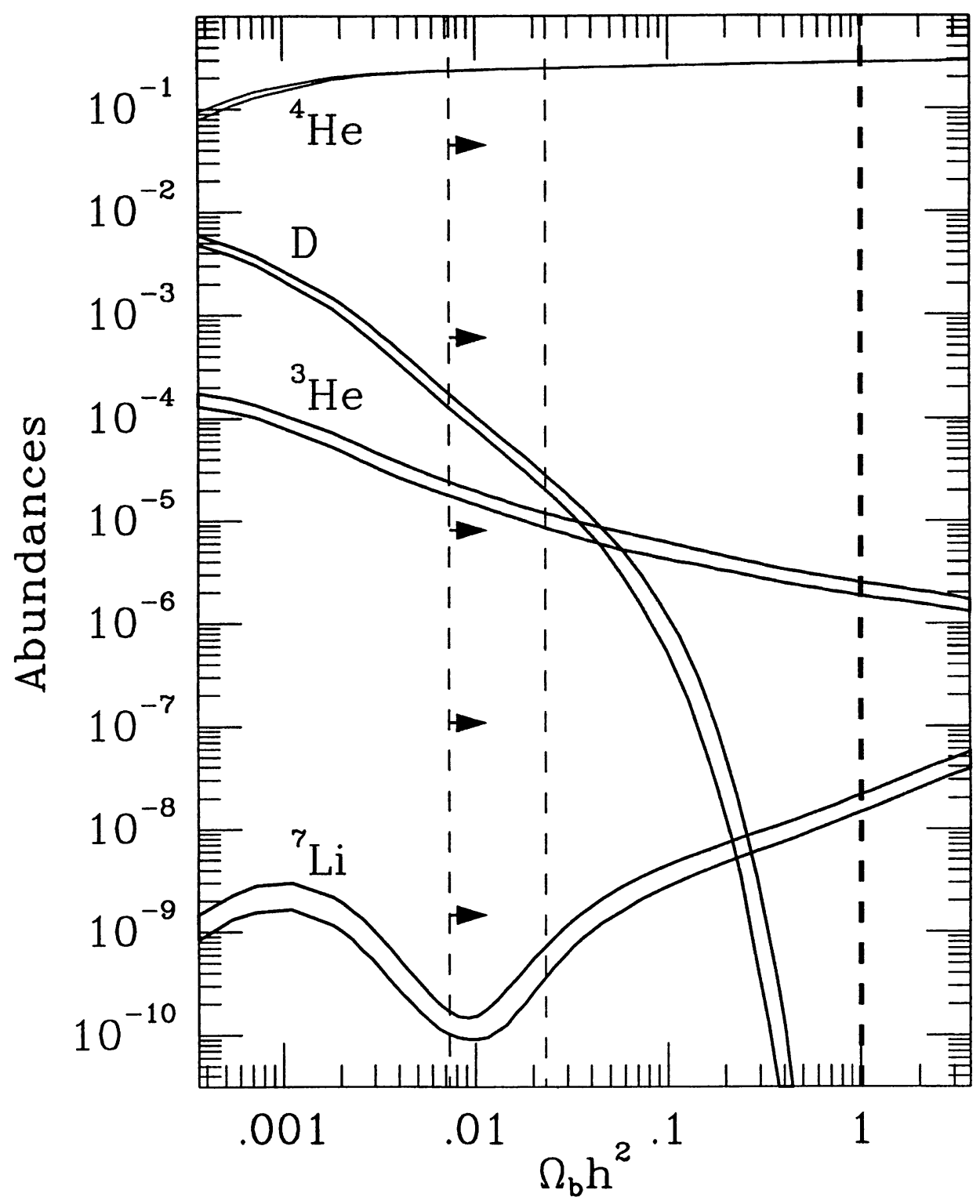

Figure 1. Big Bang Nucleosynthesis abundance yields versus baryon density $\left(\Omega_{b}\right)$ and $\eta \equiv \frac{n_{b}}{n_{\gamma}}$ for a homogeneous universe. ( $h \equiv H_{0} / 100 \mathrm{~km} / \mathrm{sec} / \mathrm{Mpc}$; thus, the concordant region of $\Omega_{b} h^{2} \sim 0.015$ corresponds to $\Omega_{b} \sim 0.06$ for $H_{0}=50 \mathrm{~km} / \mathrm{sec} / \mathrm{Mpc}$.) Figure is from Copi, Schramm and Turner (1994). Note concordance region is slightly larger than Walker et al. (1991) due primarily to inclusion of possible systematic errors on $\mathrm{Li} / \mathrm{H}$. The width of the curves represents the uncertainty due to input of nuclear physics in the calculation. Recent measurements by Tytler, Fan and Burles (1996) narrow the vertical concordance region towards the high $\Omega_{b}$ side. 
atively simple by the standards of explosive nucleosynthesis calculations in supernovae, with the changes over the last 25 years being mainly in terms of more recent nuclear reaction rates as input, not as any great calculational insight, although the current Kawano code (Kawano et al., 1988) is somewhat streamlined relative to the earlier Wagoner code[6]. In fact, the earlier Wagoner code (Wagoner et al., 1967) is, in some sense, a special adaptation of the larger nuclear network calculation developed by Truran (1965; Truran et al., 1966) for work on explosive nucleosyntheis in supernovae. With the exception of $\mathrm{Li}$ yields and non-yields of $\mathrm{Be}$ and $\mathrm{B}$ (Steigman et al., 1993), the reaction rate changes over the past 25 years have not had any major affect (see Yang et al., 1984) and Krauss and his collaborators (Krauss and Romanelli, 1990; Kernan and Krauss, 1994), or Copi, Schramm, and Turner (1994) for a discussion of uncertainties). The one key improved input is a better neutron lifetime determination (Mampe et al., 1989; Mampe et al., 1993). There has been much improvement in the $\mathrm{t}(\alpha, \gamma){ }^{7} \mathrm{Li}$ reaction rate, but as the width of the curves in Figure 1 shows, the ${ }^{7} \mathrm{Li}$ yields are still the poorest determined, both because of this reaction and even more because of the poorly measured ${ }^{3} \mathrm{He}(\alpha, \gamma){ }^{7} \mathrm{Be}$.

With the exception of the effects of elementary particle assumptions, to which we will return, the real excitement for BBN over the last 25 years has not really been in redoing the basic calculation. Instead, the true action is focused on understanding the evolution of the light element abundances and using that information to make powerful conclusions. In the 1960's, the main focus was on ${ }^{4} \mathrm{He}$ which is very insensitive to the baryon density. The agreement between $\mathrm{BBN}$ predictions and observations helped support the basic Big Bang model but gave no significant information, at that time, with regard to density. In fact, in the mid-1960's, the other light isotopes (which are, in principle, capable of giving density information) were generally assumed to have been made during the T-Tauri phase of stellar evolution (Fowler et al., 1962), and so, were not then taken to have cosmological significance. It was during the 1970's that BBN fully developed as a tool for probing the universe. This possibility was in part stimulated by Ryter et al. (1970) who showed that the T-Tauri mechanism for light element synthesis failed. Furthermore, D abundance determinations improved significantly with solar wind measurements (Geiss and Reeves, 1971; Black, 1971) and the interstellar work from the Copernicus satellite (Rogerson and York, 1973). (Recent HST observations reported by Linsky et al. (1993) have compressed the local ISM D error bars considerably.) Reeves, Audouze, Fowler and Schramm (1973) argued for cosmological D and were able to place a constraint on the baryon density excluding a universe closed with baryons. Subsequently, the D arguments were cemented when Epstein, Lattimer and Schramm (1976) proved that no realistic astrophysical process 
other than the Big Bang could produce significant D. This baryon density was compared with dynamical determinations of density by Gott, Gunn, Schramm and Tinsley (1974). See Figure 2 for an updated $H_{0}-\Omega$ diagram.

In the late 1970's, it appeared that a complimentary argument to D could be developed using ${ }^{3} \mathrm{He}$. In particular, it was argued (Rood et al., 1976) that, unlike $\mathrm{D},{ }^{3} \mathrm{He}$ was made in stars; thus, its abundance would increase with time. Unfortunately, recent data on ${ }^{3} \mathrm{He}$ in the interstallar medium (Gloeckler and Geiss, 1996) has shown that ${ }^{3} \mathrm{He}$ has been constant for the last $5 \mathrm{Gyr}$. Thus, low mass stars are not making a significant addition, contrary to these previous theoretical ideas. Furthermore, Rood, Bania and Wilson (1992) have shown that interstellar ${ }^{3} \mathrm{He}$ is quite variable in the Galaxy, contrary to expectations for a low mass star-dominated nucelus. However, the work on planetary nebulae shows that at least some low mass stars do produce ${ }^{3} \mathrm{He}$. Nonetheless, the current observational situation clearly shows that arguments based on theoretical ideas about ${ }^{3} \mathrm{He}$ evolution should be avoided (c.f. Hata et al. (1995) where their "crisis" is really about ${ }^{3} \mathrm{He}$ problems [and excessively small assumed uncertainties in $\left.{ }^{4} \mathrm{He}\right]$, not $\left.\left.\mathrm{BBN}\right]\right)$. Since ${ }^{3} \mathrm{He}$ now seems not to have a well understood history, simple ${ }^{3} \mathrm{He}$ or ${ }^{3} \mathrm{He}+\mathrm{D}$ inventory arguments are misleading at best. However, one is not free to go to arbitrary low baryon densities and high primordial $\mathrm{D}$ and ${ }^{3} \mathrm{He}$, since processing of $\mathrm{D}$ and ${ }^{3} \mathrm{He}$ in massive stars also produces metals which are constrained (Copi et al., 1995; Scully et al., 1996) by the total metal content observed in the hot intra-cluster gas, if not the Galaxy. In the near future, this problem with ${ }^{3} \mathrm{He}$ evolution will be severely constrained by the extragalctic $\mathrm{D} / \mathrm{H}$. In particular, the Tytler and Burles $\mathrm{D} / \mathrm{H}=3.4 \pm 0.3 \times 10^{-5}$ (Burles \& Tytler 1997, Burles 1997) is somewhat higher than the pre-solar $\mathrm{D} / \mathrm{H}=2.4 \pm 0.4 \times 10^{-5}$ (Geiss, 1997) and about a factor of 2 above the current interstellar $\mathrm{D} / \mathrm{H}=1.5 \pm 0.1 \times 10^{-5}$ (Linsky, 1997). This tells us that the production of the current metal content of the Galaxy did not destroy more than $50 \%$ of the primordial D. Such low destruction could be accomplished with either Galactic infall or with a different stellar initial mass function at earlier times.

It was interesting that the abundances of the other light elements led to the requirement that ${ }^{7} \mathrm{Li}$ be near its minimum of ${ }^{7} \mathrm{Li} / \mathrm{H} \sim 10^{-10}$, which was verified by the Pop II Li measurements of Spite and Spite (1982; Rebolo et al., 1988; Hobbs and Pilachowski, 1988), hence yielding the situation emphasized by Yang et al. (1984) that the light element abundances are consistent over nine orders of magnitude with BBN, but only if the cosmological baryon density, $\Omega_{b}$, is constrained to be around $6 \%$ of the critical value (for $H_{0} \simeq 50 \mathrm{~km} / \mathrm{sec} / \mathrm{Mpc}$ ). The Li plateau argument was further strengthened with the observation of ${ }^{6} \mathrm{Li}$ in a Pop II star by Smith, Lambert and Nissen (1993). Since ${ }^{6} \mathrm{Li}$ is much more fragile than ${ }^{7} \mathrm{Li}$, and yet it 


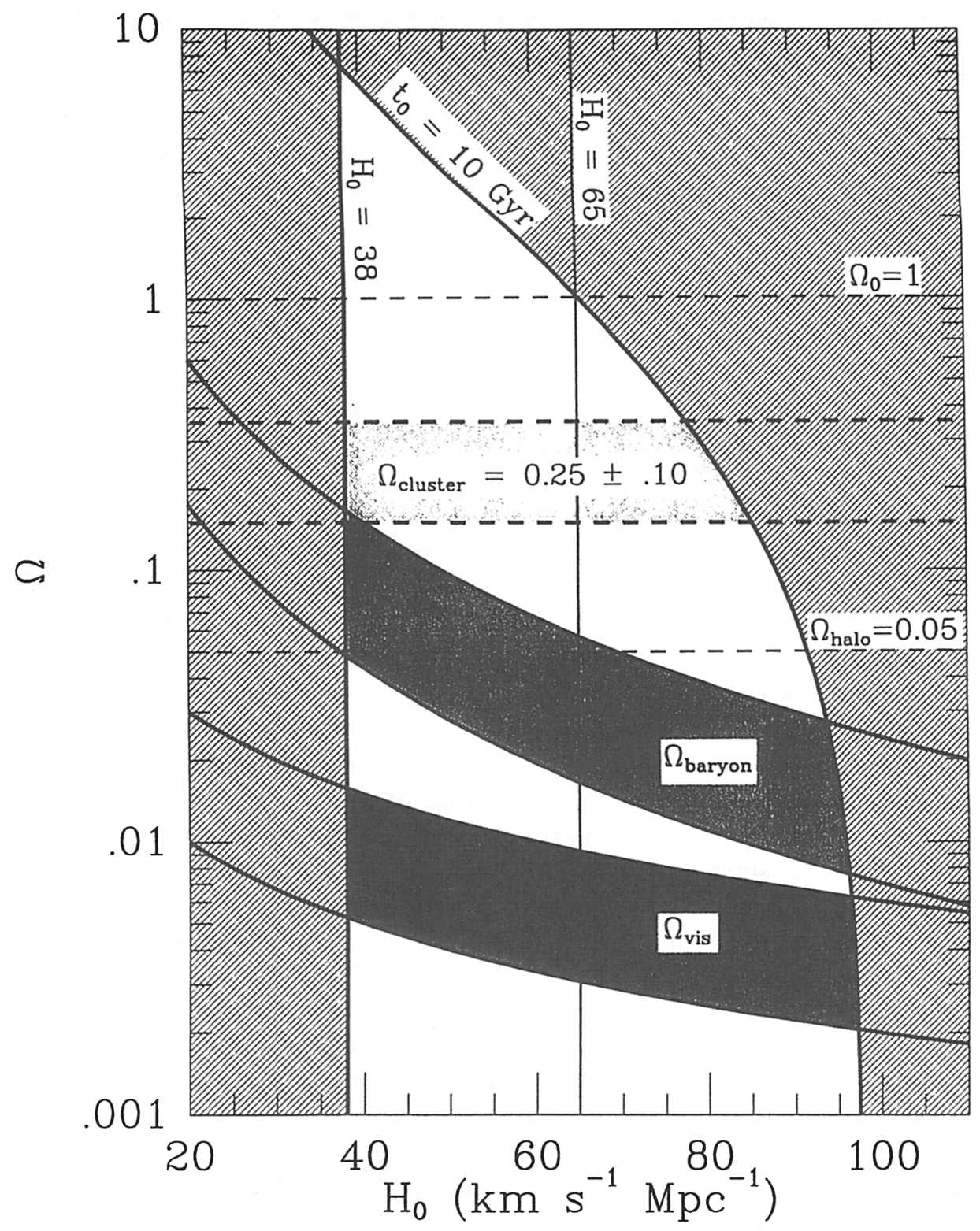

Figure 2. An updated version of $H_{0}-\Omega$ diagram of Gott, Gunn, Schramm and Tinsley (1974) showing that $\Omega_{b}$ does not intersect $\Omega_{V I S I B L E}$ for any value of $H_{0}$ and that $\Omega_{\text {TOTAL }}>0.1$, so non-baryonic dark matter is also needed (Shi et al., 1995).

survived, no significant nuclear depletion of ${ }^{7} \mathrm{Li}$ is possible (Steigman et al., 1993; Olive and Schramm, 1992; Lemoine et al., 1997). This observation of ${ }^{6} \mathrm{Li}$ was verified by Hobbs and Thorburn (1994). Lithium depletion mecha- 
nisms are also severly constrained by the recent work of Spite et al. (1996) showing that the lithium plateau is also found in Pop II tidally locked binaries. Thus, meridonal mixing is not causing significant lithium depletion. Recently Nollett et al. (1997) have discussed how ${ }^{6} \mathrm{Li}$ itself might eventually become another direct probe of BBN depending on the eventual low energy measurement of the $\mathrm{D}(\alpha \gamma){ }^{6} \mathrm{Li}$ cross section and on spectroscopy improvements for extreme metal-poor dwarfs. With the new extragalactic $\mathrm{D} / \mathrm{H}$, one should be able to turn the ${ }^{7} \mathrm{Li}$ argument around and argue how much depletion and/or what model atmosphere is necessary. It is again clear from this argument that large amounts of depletion did not occur, contrary to the earlier models of Delyannis (1995).

Another development back in the 70's for BBN was the explicit calculation of Steigman, Schramm and Gunn (1977) showing that the number of neutrino generations, $N_{\nu}$, had to be small to avoid overproduction of ${ }^{4}$ He. (Earlier work (Tayler and Hoyle, 1963; Schvartzman, 1969; Peebles, 1971) had commented about a dependence on the energy density of exotic particles but had not done an explicit calculation probing $N_{\nu}$.) To put this in perspective, one should remember that the mid-1970's also saw the discovery of charm, bottom and tau, so that it almost seemed as if each new detector produced new fundamental particle discoveries, and yet, cosmology was arguing against this "conventional" wisdom. Over the years, the limit on $N_{\nu}$ improved with ${ }^{4} \mathrm{He}$ abundance measurements, neutron lifetime measurements, and with limits on the lower bound to the baryon density, hovering at $N_{\nu} \lesssim 4$ for most of the 1980's and dropping to slightly lower than 4 just before LEP and SLC turned on (Walker et al., 1991; Olive et al., 1990; Schramm and Kawano, 1989; Pagels, 1991). This was verified by the results of the LEP Collaborations (1992; see also the CERN preprint CERN-PPE/96-183) where now the overall average is $N_{\nu}=2.989 \pm 0.012$. A recent examination of the cosmological neutrino limit by Copi et al. (1997) in the light of the recent ${ }^{3} \mathrm{He}$ and $\mathrm{D} / \mathrm{H}$ work shows that the $\mathrm{BBN}$ limit remains between 3 and 4 for all reasonable assumption options. It should be noted that this limit remains robust despite the uncertainties on ${ }^{4} \mathrm{He}$ systematics, since those uncertainties are still relatively small compared to a $\Delta \mathrm{N}_{\nu}$ of unity, although they are not small compared to significant shifts in ${ }^{4} \mathrm{He}$ implications for $\Omega_{b}$.

The recent apparent convergence of the extra-galactic $\mathrm{D} / \mathrm{H}$ measurements towards the lower values (Burles and Tytler, 1997; Hogan, 1998) $\mathrm{D} / \mathrm{H} \sim 3 \times 10^{-5}$ is beginning to collapse the $\Omega_{B}$ band in Figure 1 to a relatively narrow strip on the high $\Omega_{B}$ side (see arrows). However, such a full collapse at present is probably a bit premature. For example, Webb et al. (1997) find another high value of $\mathrm{D}$ and Cowie (1997) and his collegues still argue for possible high values. In any case, it is clear that deuteronomy 
(the study of deuterium in the cosmos) is a success since: 1) deuterium is clearly cosmological as it is seen in low metalicity and high redshift Lyman$\alpha$ clouds; 2 ) the primordial $\mathrm{D} / \mathrm{H}$ is higher than the present ISM $\mathrm{D} / \mathrm{H}$, as predicted by theory; and 3) the range of values for primordial $\mathrm{D} / \mathrm{H}$, regardless of whether or not the high or low ones win out, is consistent with the range of expectations based on the other light nuclei.

One potential problem that the low $\mathrm{D} / \mathrm{H}$, high $\Omega_{B}$ solution raises is the fact that the central primordial ${ }^{4} \mathrm{He}$ mass fraction is $\sim 0.23$, rather than $\sim 0.245$, which the Tytler and Burles (Tytler et al., 1996; Tytler, 1997; Burles and Tytler 1997) D/H value would prefer for concordance. However, as Copi et al. (1997) emphasize, systematic uncertainties in $\mathrm{Y}_{p}$ cannot rule out such an excursion. But clearly we have to look carefully at ${ }^{4} \mathrm{He}$. The recent work of Thuan et al. (1996) on $y \sim 0.24$ shows how uncertain the present situation is, but the resolution remains to be found. How high $Y_{p}$ can be and still be consistent with the He observations in extragalactic $\mathrm{H}-\mathrm{II}$ regions is still quite debatable, although most agree that $Y_{p}$ up to 0.25 is not impossible. Schramm and Turner (1998) show that a Bayesian analysis of the plausible upper limit on $\mathrm{Y}_{p}$ centers on 0.25 .

The power of homogeneous BBN comes from the fact that essentially all of the physics input is well determined in the terrestrial laboratory. The appropriate temperature regimes, 0.1 to $1 \mathrm{MeV}$, are well explored in nuclear physics laboratories. Thus, what nuclei do under such conditions is not a matter of guesswork, but is precisely known. In fact, it is known for these temperatures far better than it is for the centers of stars like our Sun. The center of the Sun is only a little over $1 \mathrm{keV}$, thus, below the energy where nuclear reaction rates yield significant results in laboratory experiments, and only the long times and higher densities available in stars enable anything to take place.

\section{Density of Baryons}

The bottom line that emerges from the above discussion is that (Copi et al., 1997)

$$
0.01 \leq \Omega_{B} h_{0}^{2} \leq 0.025
$$

where $h_{0} \equiv H_{0} / 100 \mathrm{~km} / \mathrm{sec} / \mathrm{Mpc}$. If the Tytler arguments on $\mathrm{D} / \mathrm{H}$ do indeed hold up, then this will compress towards the high side, say $\Omega_{B} h^{2} \sim$ $0.02 \pm 0.005$. Let us now compare with other ways of estimating $\Omega_{b}$.

Attempts to circumvent the conclusion of homogeneous BBN by invoking a first-order quark-hadron phase transition (Applegateet al., 1988; Alcock et al., 1987) have merely illustrated the robustness of the conclusions. Figure 3 illustrates this fact, showing that for an optimized first-order quark-hadron phase transition, the abundances are only fit for the same 


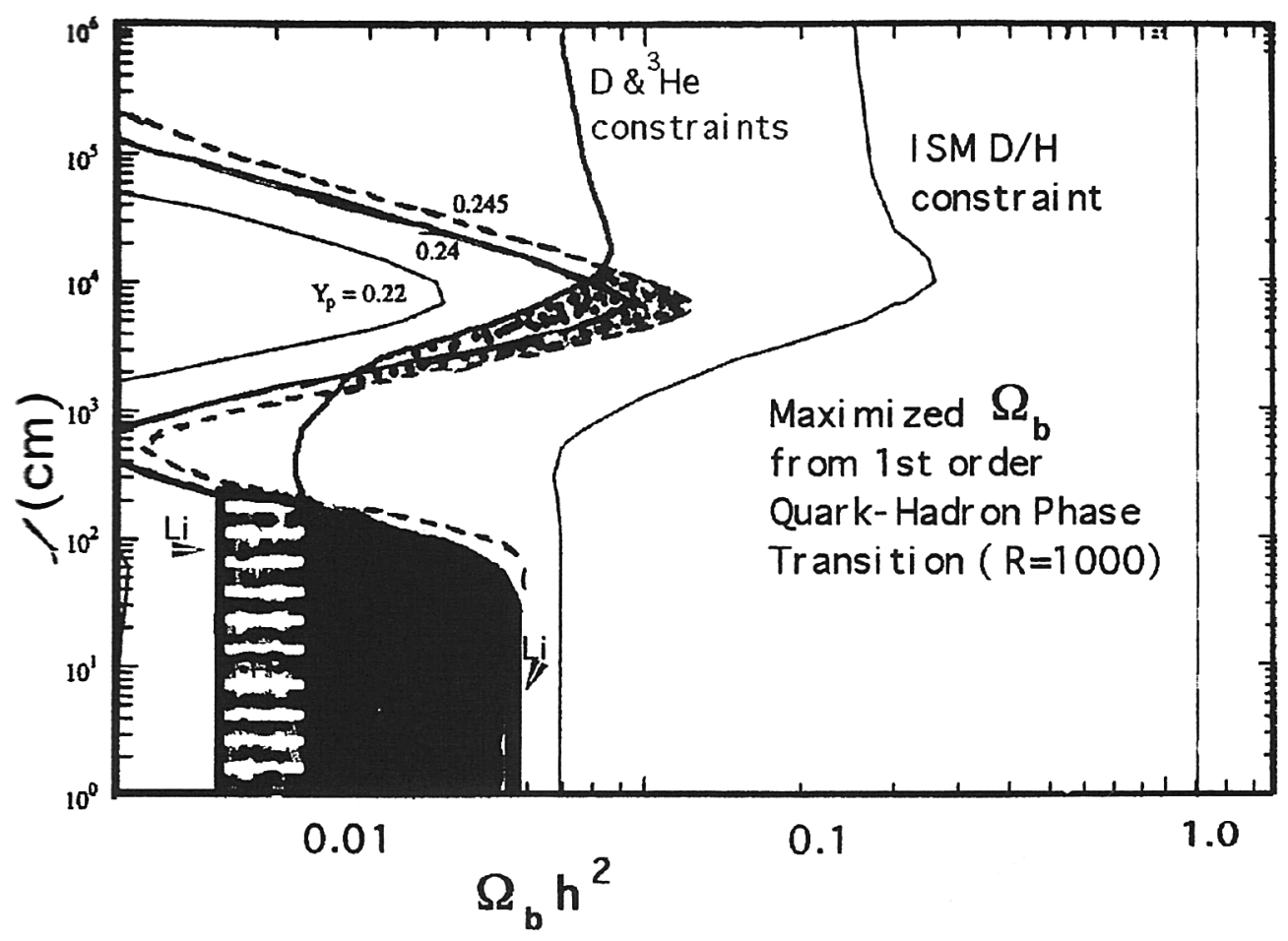

Figure 3. An assumed first order quark-hadron phase transition still yields the same allowed range on $\Omega_{b} h^{2}$ when the same abundance constraints are used. The parameter $l$ is the separation of nucleation sites (the most sensitive additional parameter in the calculation). The dotted region is an additional allowed region if lithium is completely ignored. Even then, the constraint on $\Omega_{b}$ is within a factor of 2 of the standard value.

range in $\Omega_{b} h^{2}$ as in the homogeneous case. Only if the lithium constraint is completely ignored can higher $\Omega_{b} h^{2}$ values work, and even then, only a factor of 2 is possible.

\subsection{LYMAN- $\alpha$ CLOUDS}

Recent work by Bi and Davidsen (1997), by Quashnock and Vanden Berk (1997), and by Weinberg et al. (1997) also argues that the density of gas in the form of Lymon- $\alpha$ clouds at high redshift is consistent with the high end of the Big Bang Nucleosynthesis range on $\Omega_{b}$. This would appear to resolve the long time problem of where are the "dark baryons." It is well known that $\Omega_{V I S I B L E} \lesssim 0.01$, which, when compared to $\Omega_{B B N}$, implies that the bulk of the baryons are not associated with stellar material. At least at high redshift this unseen material appears to have been found in these Lyman- $\alpha$ clouds. In conjunction with the Lyman- $\alpha$ clouds, it should also be noted that singly ionized helium is seen in the intergalactic gas, thus supporting 
the BBN fact that helium is primordial, and also supporting the point that significant numbers of baryons were between galaxies at high redshift (Jakobsen et al., 1994; Bi and Davidsen, 1997).

\subsection{HOT GAS IN CLUSTERS}

Hot gas has been found in clusters of galaxies by ROSAT and ASCA. The temperature of the gas can be used to estimate the gravitational potential of the clusters if it is assumed that the gas is vivialized and purely supported by thermal pressure. Similarly, the intensity of the emission can be used to estimate the density of the gas. White et al. (1993) have shown that the typical values for $\mathrm{x}$-ray clusters yield a hot gas to total mass ratio $M_{H O T} / M_{\text {TOT }}$ of about 0.2 .

Cluster masses can be estimated either from the temperature of the hot gas or from dynamics or from gravitational lensing. All yield the cluster implied density, $\Omega_{C L U S T E R}$, of $\sim 0.25 \pm 0.10$. Thus, the implied bayon density from x-ray gas in clusters, $\Omega_{b, C L U S T E R} \simeq \frac{M_{\text {HOT }}}{M_{T O T}} \times \Omega_{C L U S T E R} \sim$ 0.05 , in good agreement with the $\mathrm{BBN}$ value for $\mathrm{H}=50$ and for the Tytler $\mathrm{D} / \mathrm{H}$.

\subsection{SUNYAEV-ZELDOVICH EFFECT}

Although not completed independent of the above x-ray gas determination, reliable maps of clusters are now being made using the Sunyaev-Zeldovich technique of looking for the distortion in the microwave background due to compton scattering from the hot, $\mathrm{x}$-ray producing gas in the clusters (Carlstrom, 1998). The baryon density determined in this way has an $\mathrm{h}^{-1}$ dependence, whereas the pure $\mathrm{x}$-ray gas determination goes as $\mathrm{h}^{-3 / 2}$ (and $\mathrm{BBN}$ as $\mathrm{h}^{-2}$ ). Carlstrom (private conversation, 1997) finds $\Omega_{b} \sim 0.06 \pm$ $02 h^{-1}$ which is consistent with BBN for $\mathrm{h} \sim 0.6$.

\subsection{MICROWAVE ANISOTROPIES}

The method with the most potential for checking $\Omega_{b}$ is the measurement of the acoustic peaks in the microwave background anisotropy at angular scales near $1^{\circ}$ or less (Jungman et al., 1996a; 1996b). The height of the first doppler (acoustic) peak for gaussian fluctuation models is directly related to $\Omega_{B} h^{2}$, thus a direct check on BBN. Current experiments at the South Pole, at Sasketoon, and using balloons seem to favor values near the high side of the $\mathrm{BBN}$ range, but the present uncertainties are too large to make any strong statements. However, the next generation of satellites, NASA's MAP and ESA's PLANCK (formerly COBRAS/SAMBA) should be able 
to fix $\Omega_{b} h^{2}$ to better than $10 \%$ (if the sky is gaussian), which should provide a dramatic test of Big Bang Nucleosynthesis.

\section{Dark Matter}

The robustness of the basic BBN arguments and the new $\mathrm{D} / \mathrm{H}$ measurements have given renewed confidence to the limits on the baryon density constraints. Let us convert this density regime into units of the critical cosmological density for the allowed range of Hubble expansion rates. This is shown in Figure 2. Figure 2 also shows the lower bound on the age of the universe of $10 \mathrm{Gyr}$ from both nucleochronology and from globular cluster dating (Shi et al., 1995) and a lower bound on $H_{0}$ of 38 from extreme type IA supernova models with pure $1.4 \mathrm{M}_{\odot}$ carbon white dwarfs being converted to ${ }^{56} \mathrm{Fe}$. The constraint on $\Omega_{b}$ means that the universe cannot be closed with baryonic matter. (This point was made over twenty years ago (Reeves et al., 1970) and has proven to be remarkably strong.) If the universe is truly at its critical density, then nonbaryonic matter is required. This argument has led to one of the major areas of research at the particle-cosmology interface, namely, the search for non-baryonic dark matter. In fact, from the lower bound on $\Omega_{\text {TOTAL }}$ from cluster dynamics of $\Omega_{T O T A L}>0.15$, it is clear that non-baryonic dark matter is required unless $H_{0}<40$, regardless of whether $\Omega_{T O T A L}=1$. Figure 2 also shows the range of $\Omega_{V I S I B L E}$ and shows that there is no overlap between $\Omega_{b}$ and $\Omega_{V I S I B L E}$. Hence, the bulk of the baryons are dark, that is, not in the form of stars.

Another interesting conclusion (Gott et al., 1974) regarding the allowed range in baryon density is that it is in agreement with the density implied from the dynamics of single galaxies, including their dark halos. The recent MACHO (Alcock et al., 1993) and (Aubourg et al., 1993) reports of halo microlensing may well indicate that at least some of the dark baryons are in the form of baryonic objects in the halo. However, Gates, Gyuk and Turner (1995), and Alcock et al. $(1997,1993)$ show that the observed distribution of MACHOs favors less than $50 \%$ of the halo being in the form of MACHOS, but a $100 \%$ MACHO halo cannot be completely excluded, yet.

For dynamical estimates of $\Omega$, one estimates the mass from $M \sim \frac{v^{2} r}{G}$ where $v$ is the relative velocity of the objects being studied, $r$ is their separation distance, and $G$ is Newton's constant. The proportionality constant out front depends on orientation, relative mass, etc. For large systems such as clusters, one uses averaged quantities. For single galaxies $v$ would represent the rotational velocity and $r$, the radius of the star or gas cloud. It is this technique which yields the cluster bound on $\Omega$ shown in Figure 2. As mentioned above, the value of $\Omega_{C L U S T E R} \sim 0.25$ is also obtained in those few cases where alignment produces giant gravitational-lens arcs (Fahlman 
et al.,1994) and also from the gravitational potential implied by the temperature of the observed $x$-ray gas in the clusters. As Davis (1997) showed, if the large scale velocity flows measured from the IRAS survey are due to gravity, then $\Omega_{I R A S} \gtrsim 0.2$. For $H_{0}>40, \Omega_{C L U S T E R}$ already requires $\Omega_{\text {TOTAL }}>\Omega_{\text {BARYON }}$ and hence the need for non-baryonic dark matter.

An $\Omega$ of unity is, of course, preferred on theoretical grounds since that is the only long-lived natural value for $\Omega$, and inflation (Guth, 1981; Linde, 1982) or something like it provided the early universe with the mechanism to achieve that value and thereby solve the flatness and smoothness problems. Note that our need for exotica is not dependent on the existence of dark galatic halos and that high values of $H_{0}$ increase the need for non-baryonic dark matter.

It is also interesting to note that the convergence of $\Omega$ on cluster scales at $\sim 0.25 \pm 0.1$ has important implications. If $\Omega_{T O T A L}$ is really unity, it would necessitate clusters not being a fair sample of the universe. Since standard CDM implies cluster scales as fair samples, this would imply a more complex structure formation picture. Options include biasing on cluster scales, a very hot dark matter component, or even a smooth background component such as a $\Lambda_{0}$ term, or a vacuum energy from a late-time phase transition (Hill and Schramm, 1985; Frieman et al., 1996).

\section{Acknowledgments}

I would like to thank my collaborators, Craig Copi, Ken Nollett, Martin Lemoine, David Dearborn, Brian Fields, Dave Thomas, Gary Steigman, Brad Meyer, Keith Olive, Angela Olinto, Bob Rosner, Michael Turner, George Fuller, Sean Scully, Karsten Jedamzik, Rocky Kolb, Grant Mathews, Bob Rood, Jim Truran and Terry Walker for many useful discussions. I would further like to thank Art Davidsen, Poul Nissen, Jeff Linsky, David Tytler, Len Cowie, Scott Burles, Craig Hogan, Julie Thorburn, Doug Duncan, Lew Hobbs, Evan Skillman, Bernard Pagel and Don York for valuable discussion regarding the astronomical observations.

This work is supported by the NASA and the DoE (nuclear) at the University of Chicago, and by the DoE and by NASA grant NAG5-2788 at Fermilab.

\section{References}

Alcock, C. et al. (1993) Nature Vol. no. 365, 621-623.

Alcock, C. et al. (1997) Astrophys. J. Vol. no. 486, 697-726.

Alcock, C.R., Fuller, G. and Mathews, G. (1987) Astrophys. J. Vol. no. 320, 439-447.

Alpher, R.A., Bethe, H. and Gamow, G. (1948) Phys. Rev., Vol. no. 73, 803-804.

Alpher, R.A., Follin, J.W. and Herman, R.C. (1953) Phys. Rev., Vol. no. 92, 1347-1361. 
Applegate, J.H., Hogan, C.J. and Scherrer, R.J. (1988) Astrophys. J. Vol. no. 329, 572-579.

Aubourg, E. et al. (1993) Nature Vol. no. 365, 623-625.

Bi, H.G. and Davidsen, A.F. (1997) Astrophys. J. Vol. no. 479, 523-542.

Black, D. (1971) Nature Vol. no. 234, 148-149.

Burles, S. (1997) Ph.D. Thesis, University of California at San Diego.

Burles, S. and Tytler, D. (1997) Astrophys. J., submitted.

Carlstrom, J.E., Greco, L., Holzapfel, W.L. and Joy, M. (1998) in ed. A. Olinto, Proc. of the 18th Texas Symposium on Relativisitic Astrophysics, in press (World Scientific, Singapore).

Copi, C.J., Schramm, D.N. and Turner, M.S. (1994),Science Vol. no. 267, (192-(199.

Copi, C.J., Schramm, D.N. and Turner, M.S. (1995) Astrophys. J. Vol. no. 455, L95-L98.

Copi, C.J., Schramm, D.N. and Turner, M.S. (1997) Phys. Rev. D Vol. no. 55, 33893393.

Davis, M. (1997) in A. Olinto (ed.). Proc. of the 18th Texas Symposium on Relativisitic Astrophysics, in press (World Scientific, Singapore).

Delyannis, C.P. (1995) in The Light Element Abundances (Proc. of an ESO/EIPC Workshop held in Marciana Marina, Isola d'Elba, May 1994, ed. P. Crane, ESO Astrophysics Symposia (Springer-Verlag, Berlin), 395-409.

Epstein, R., Lattimer, J. and Schramm, D.N. (1976) Nature Vol. no. 263, 198-202.

Fahlman, G. Kaiser, N., Squires, G. and Woods, D. (1994) Astrophys. J. Vol. no. 437, 56-62.

Fowler, W.A., Greenstein, J. and Hoyle, F. (1962) Geophys. J.R.A.S. Vol. no. 6, 148-220.

Frieman, J.A., Hill, C.T., Stebbins, A. and Waga, I. (1996) Phys. Rev. Lett. Vol. no. 75, 2077-2080.

Gates, E., Gyuk, G. and Turner, M. (1995) Phys. Rev. Lett. Vol. no. 74, 3724-3727.

Geiss, J. (1997) in ed. C. de Jaeger, Proc. of International Space Science Institute Workshop on "Primordial Nuclei and Their Galactic Evolution," Bern, Switzerland, May (1997, Space Sciences Series of ISSI, in press (Kluwer Academic, Dordrecht).

Geiss, J. and Reeves, H. (1971) Astron. and Astrophys. Vol. no. 18, 126-132.

Gloeckler, G. and Geiss, J. (1996) Nature Vol. no. 381, 210-212.

Gott, J.R., III, Gunn, J., Schramm, D.N. and Tinsley, B.M. (1974) Astrophys. J. Vol. no. $(194,543-553$.

Guth, A. (1981) Phys. Rev. D Vol. no. 23, 347-356.

Hata, N., Scherrer, R.J., Steigman, G., Thomas, D., Walker, T.P., Bludman, S. and Langacker, P. (1995) Phys. Rev. Lett. Vol. no. 75, 3977-3980.

Hayashi, C. (1950) Prog. Theor. Phys. Vol. no. 5, 224-235.

Hill, C.T. and Schramm, D.N. (1985) Phys. Rev. D Vol. no. 31, 564-580.

Hobbs, L. and Pilachowski, C. (1988) Astrophys. J. 326, L23-L26.

Hobbs, L. and Thorburn, J. (1994) Astrophys. J. Lett. 428, L25-L28.

Hogan, C. (1998) in ed. A. Olinto, Proc. of the 18th Texas Symposium on Relativisitic Astrophysics, in press (World Scientific, Singapore).

Jakobsen, P., Boksenberg, A., Deharveng, J.M., Greenfield, P., Jedrzewski, R. and Paresce, F. (1994) Nature Vol. no. 370, 35-39.

Jungman, G., Kosowsky, A., Kamionkowski, M., Spergel, D.N. (1996a) Phys. Rev. Lett. Vol. no. 76, 1007-1010.

Jungman, G., Kosowsky, A., Kamionkowski, M., Spergel, D.N. (1996b) Phys. Rev. D Vol. no. 54, 1332-1344.

Kawano, L., Schramm, D.N. and Steigman, G. (1988) Astrophys. J. Vol. no. 327, 750754.

Kernan, P. and Krauss, L. (1994) Phys. Rev. Lett. 72, 3309-3312.

Krauss, L.M. and Romanelli, P. (1990) Astrophys. J. Vol. no. 358, 47-59.

Kronberg, P.P. (1994) Rep. Prog. Phys. Vol. no. 57, 325-382.

Lemoine, M, Schramm, D.N., Truran, J.W. and Copi, C.J. (1997) Astrophys. J. Vol. no. 478, 554-562. 
The LEP Collaborations: ALEPH, DELPHI, L3, and OPAL (1992) Phys. Lett. B Vol. no. 276, 247-253.

Linde, A. (1990) Particle Physics and Inflationary Cosmology (Harwood Academic Publishers, N.Y.).

Linsky, J. (1997) in Proc. of International Space Science Institute Workshop on "Primordial Nuclei and Their Galactic Evolution," Bern, Switzerland, May (1997, Space Sciences Series of ISSI, ed. C. de Jaeger, in press (Kluwer Academic, Dordrecht).

Linsky, J., Brown, A., Gayley, K., Diplas, A., Savage, B., Ayres, T., Landsman, W., Shore, S. and Heap, S. (1993) Astrophys. J. Vol. no. 402, 694-709.

Mampe, W. et al. (1993) JETP Lett. Vol. no. 57, 82-87.

Mampe, W., Ageron, P., Bates, C., Pendlebury, J.M. and Steyerl, A. (1989) Phys. Rev. Lett. Vol. no. 63A, 593-596.

Mushotsky, R. (1993) in eds. C.W. Akerlof and M.A. Srednicki, Relativistic Astrophysics and Particle Cosmology Texas PASCOS 92, Annals of the N.Y Academy of Sciences Vol. no. 688, 184-194.

Nollett, K., Lemoine, M. and Schramm, D.N. (1997) Phys. Rev. C. Vol. no. 56, 11441151.

Olive, K. and Schramm, D.N. (1992) Nature Vol. no. 360, 439-442.

Olive, K., Schramm, D.N., Steigman, G., Turner, M. and Yang, J. (1981) Astrophys. J. Vol. no. 246, 557-568.

Olive, K., Schramm, D.N., Steigman, G. and Walker, T. (1990) Phys. Lett. B Vol. no. 236, 454-460.

Pagel, B. (1991) Physica Scripta, Vol. no. T36, 7-15.

Peebles, P.J.E. (1966) Phys. Rev. Lett. Vol. no. 16, 410-413.

Peebles, P.J.E. (1971) Physical Cosmology (Princeton University Press).

Quashnock, J.M. and Vanden Berk, D.E. (1997) Astrophyhs. J., submitted.

Rebolo, R., Molaro, P. and Beckman, J. (1988) Astron. and Astrophys. Vol. no. (192, (192-205.

Reeves, H., Audouze, J., Fowler, W.A. and Schramm, D.N. (1973) Astrophys. J. Vol. no. 179, 909-930.

Reeves, H., Fowler, W.A. and Hoyle, F. (1970) Nature 226, 727-729.

Rogerson, J. and York, D. (1973) Astrophys. J. Vol. no. 186, L95-L98.

Rood, R.T., Bania, T., and Wilson, J. (1992) Nature 355, 618-620.

Rood, R.T., Steigman, G. and Tinsley, B.M. (1976) Astrophys. J. Vol. no. 207, L57-

Ryter, C., Reeves, H., Gradstajn, E. and Audouze, J. (1970) Astron. and Astrophys Vol. no. 8, 389-397.

Schramm, D.N. (1997) 'Primordial Nucleosynthesis.' PNAS, Vol. no. 94, in press.

Schramm, D.N. and Kawano, L. (1989) Nuc. Inst. and Methods A Vol. no. 284, 84-88.

Schramm, D.N. and Wagoner, R.V. (1977) Ann. Rev. of Nuc. Sci. Vol. no. 27, 37-74.

Schvartzman, V.F. (1969) JETP Letters Vol. no. 9, 184-186.

Scott, D. and White, M. (1995) Gen. Rel. and Grav. Vol. no. 27, 1023-1030.

Scully, S.T., Cassé, M., Olive, K.A., Schramm, D.N., Truran, J. and Vangioni-Flam, E. (1996) Astrophys. J. Vol. no. 462, 960-968.

Shi, X., Schramm, D.N., Dearborn, D. and Truran, J.W. (1995) Comments on Astrophys. Vol. no. 17, 343-360.

Smith, V.V., Lambert, D.L. and Nissen, P.E. (1993) Astrophys. J. Vol. no. 408, 262-276.

Spite, M., Nissen, P.E. and Spite, F. (1996) Astron. and Astrophys. Vol. no. 307, 172183.

Spite, J. and Spite, M. (1982) Astron. and Astrophys. Vol. no. 115, 357-366.

Steigman, G., Fields, B.D., Schramm, D.N., Olive, K. and Walker, T. (1993) Astrophys. J. Vol. no. 415, L35-L38.

Steigman, G., Schramm, D.N. and Gunn, J. (1977) Phys. Lett. B Vol. no. 66, 202-204.

Strickland, R. and Schramm (1997) Astrophys. J., in press.

Tayler, R. and Hoyle, F. (1964) Nature Vol. no. 203, 1108-1110.

Thuan, T.X., Izotov, Y.I. and Lipovetsky, V.A. (1996) Astrophys. J. Vol. no. 463, 120- 
133.

Truran, J.W. (1965) Doctoral Thesis, Yale University.

Truran, J. W., Cameron, A.G.W. and Gilbert, A. (1966) Can. Jour. of Phys. Vol. no. 44, 563-592.

Tytler, D. (1997) in eds. N. Prantos, M. Tosi and R. von Steiger, Space Sciences Series of ISSI (Proc. of ISSI Workshop on Primordial Nuclei and their Galactic Evolution, Bern, Switzerland, May 1997, in press (Kluwer, Dordrecht), and in Space Science Reviews, ed. C. de Jaeger, in press.

Tytler, D., Fan, X.-M. and Burles, S. (1996) Nature, 381, 207.

Wagoner, R., Fowler, W.A. and Hoyle, F. (1967) Astrophys. J. Vol. no. 148, 3-49.

Walker, T., Steigman, G., Schramm, D.N., Olive, K. and Kang, H.S. (1991) Astrophys. $J$. Vol. no. 376, 51-69.

Webb, J.K., Carswell, R.F., Lanzetta, K.M., Ferlet, R., Lemoine, M., Vidal-Madjar, A. and Bowen, D.V. (1997) Nature, Vol. no. 388, 250-252.

Weinberg, D.H., Miralda-Escué, J., Hernquist, L. and Katz, N. (1997) Astrophys. J., Vol. no. 490, 564-570.

White, S.D.M., Navarro, J.F., Evrard, A.E. and Frenck, C.S. (1993) Nature Vol. no. 366, 429-433.

Yang, J., Turner, M., Steigman, G., Schramm, D.N. and Olive, K. (1984) Astrophys. J. Vol. no. 281, 493-511. 